\title{
The Comprehensive Evaluation Index System of Urban Emergency Capac- ity Based on External DEA
}

\author{
Fengxian $\mathrm{Lu}^{1,2}$, Guanghui Wang ${ }^{2, *}$, Hongbing Wang ${ }^{2}$ and and Yuan Huang ${ }^{2,3}$ \\ ${ }^{I}$ College of Environment and Planning, Collaborative Innovation Center of Three-aspect Coordination of Central-China \\ Economic Region, Henan University, Kaifeng, 475004, China: ${ }^{2}$ Institute of Policy and Management, Chinese Academy \\ of Sciences, Beijing, 100190, China, ${ }^{3}$ University of Chinese Academy of Sciences, Beijing, 100190, China
}

\begin{abstract}
Since the combined effects of the rapid urban growth and climate change, disasters such as blizzard, hurricane, earthquake and traffic jar occur in nearly every city frequently. Building an urban adaptive emergency evaluation index system is a global topic concerned by experts in recent years. It is also a top priority in current urban planning. This paper makes comprehensive research and analysis on the urban adaptive emergency capacity. Firstly, it divides the city's adaptive strength of disaster relief into tensile strength, bending strength, abrasion resistance, fatigue strength and breaking strength etc., which is the basic of building the integrated strength grade to evaluate the city's capacity of keeping out incident. Secondly, it puts forward a set of scientific, complete and quantitative assessment index system (vulnerability, elasticity, stability, rationality and plasticity etc.) for emergency capacity which is based on the during-incident emergency evaluation theory, including Mitigability Measurement, Salvability Measurement, and Recoverability Measurement. Lastly, the paper studies the comprehensive evaluation index system of urban emergency capacity based on the external DEA, which can indicate the urban adaptive capacity of disaster relief in magic cube. Above all, the results can provide the reference for urban disaster risk management.
\end{abstract}

Keywords: Adaptive emergency capacity, evaluation index system, external DEA, magic cube, urban adaptive strength.

\section{INTRODUCTION}

During the past several decades, most cities and regions in the world have been experiencing constantly growing rates of congregation of population, production and wealth, thus becoming more vulnerable and fragile when facing sudden incidents and disasters. Hence there is an urgent requirement for the innovation that provides better quantitative assessment method and techniques to evaluate the capacity of a city or region to carry incidents and disasters.

There are various kinds of analytic technique for building the urban emergency capacity in literature, but risk matrix approach (RMA) and analytic hierarchy process (AHP) has be widely applied in emergency management and the city's risk analysis. Adam S. Markowski and Quan Zhou describe a procedure for developing a fuzzy risk matrix that may be used for emerging fuzzy logic applications in different safety analyses [1-2]. Based on the deep analysis of the defects of RMA and the characteristics of emergency management, $\mathrm{Ni}$ Huihui and Chen An proposal some more extensions on risk matrix approach and demonstrated the related quondam methods (Fuzzy Risk Matrix) by a city's case study [3]. Tie Yongbo and Tang Chuan use analytic hierarchy process and Delphi method to set the weights of all evaluation indexes, and build the Evaluation Model of the urban emergency capacity from the angle of system theory [4]. Through analyzing the influencing factor of urban emergency capacity, Wang Xia and M. M. Tawan defined evaluation index of emergency capacity and three phase Model [5]. Besides, the author used the model to evaluate a city's ability to emergency.

As an important part of Modern Emergency Management, City Emergency has some characteristics. Firstly, city is the basic element of the world, which includes natural system and social system. However, the traditional emergency management strategy is mainly against natural calamity, so we should develop some new method to deal with city disaster. Chen Guo-hua and Royce Francis put forward a quantitative evaluation approach for urban and regional disaster carrying capacity (UR-DCC) from the viewpoint of disaster prevention, resistance, rescue and recovery [6-7]. Tian Yilin and Zhao Ling use Delphi to form the factor sets of evaluation index on urban emergency capacity and build the Hierarchical Diagrams of system structure based on AHP, which divides the complex evaluation index system into different factors [8-9]. These factors can visually reflect the subjection relationship of city system . Secondly, With the rapid expansion of city scale and the excessive concentration of population, some unconventional incident occur in civic centre. The single evaluation index cannot express the incident completely, so the sound urban disaster prevention index system has become a necessary arduous task. Mercedes Garcés et al. brings forward an imperative issue on how to enhance emergency management by segmenting complex influencing 


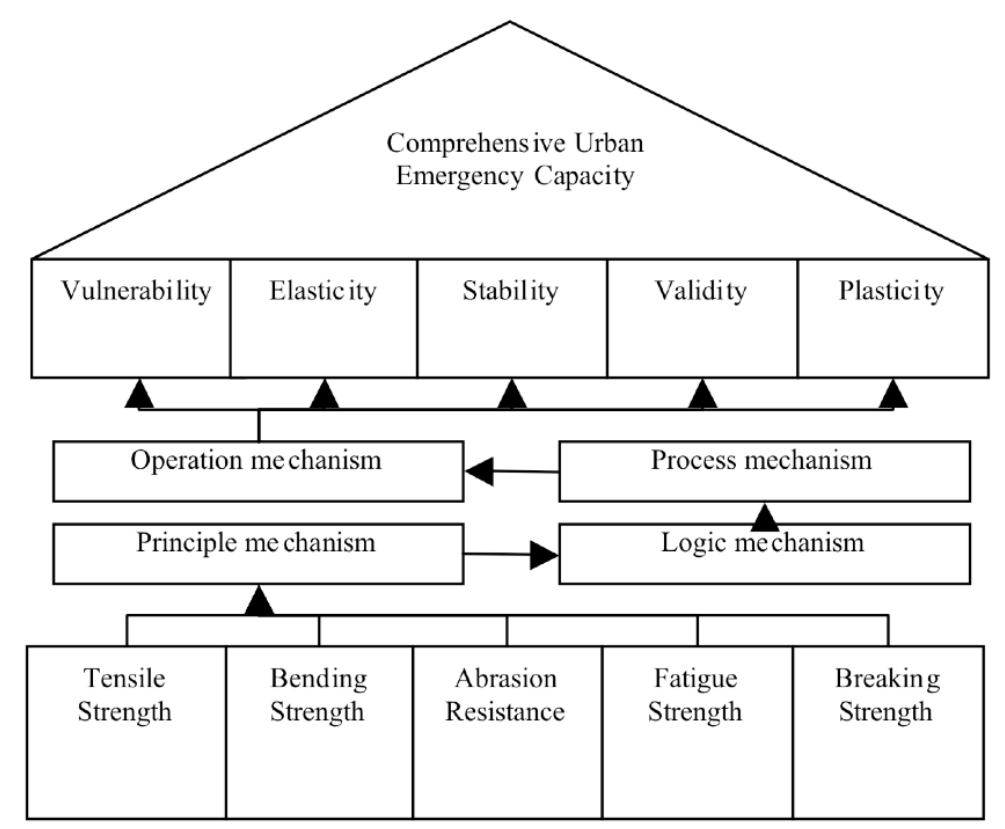

Fig. (1). The Structure of the paper.

factors into groups to improve them in a stepwise way [10]. In order to address this concern involving the vagueness of human judgments, an effective method that combines fuzzy logic and decision-making trial and evaluation laboratory (DEMATEL) is used in the paper. Jing Cheng and Jian ping Tao takes seven counties of Xiaogan, Hubei Province as the case study area [11], screens out 17 factors which closely related to drought vulnerability as evaluation index mainly from a socio-economic perspective, applies AHP to determine the weights of various relevant factors in the evaluation index, and then uses the fuzzy comprehensive evaluation method to achieve a drought vulnerability assessment.

Finally, Economic Globalization has given a boost to the international development of urban emergency rescue, such as, the assistant of global Aid agencies in dealing with the Wenchuan earthquake, the Haiti earthquake and the 2011 tsunami-earthquake in Japan. The trend of internationalization in city emergency can promote the urban adaptive capacity of disaster relief. Through a cross-country comparison between Fukui, Japan and the San Francisco Bay Area, California, USA, Kazuko Tanaka defines a new index of overall readiness to estimate the degree to which an individual's readiness for earthquakes is sufficient and appropriate [12]. The index enables a cross-country comparison of data from questionnaire surveys conducted in both seismic areas. $\mathrm{Wu}$ Xinyan and $\mathrm{Gu}$ Jianhua introduce the basic concept, the practical or immediate significance [13], the development of urban emergency management capability assessment and the existing problems at home and abroad.

In view of existing complex situation of the urban emergency capability assessment, the urban adaptive strength of disaster relief and a set of scientific, complete and quantitative assessment index system based on the whole emergency cycle were proposed. In order to reflect the city's natural and social properties, the paper extends the Applied Scope of DEA and builds the comprehensive urban emergency capacity model based on the theory of external
DEA, which is finally indicated in the form of magic cube. The rest of the paper is organized as follows. Section 2 briefly introduces the five-dimensional adaptive strength of disaster relief. Section 3 presents a set of scientific, complete and quantitative assessment index system, and describes the relationship of adaptive strength and the index system. Section 4 exploringly constructs a theoretical model of urban emergency capacity based on external DEA and the form of magic cube. Section 5 concludes this work, as shown in Fig. (1). The highlights of the paper is shown as follows.

- Define the urban adaptive strength of disaster relief: tensile strength, bending strength, abrasion resistance, fatigue strength and breaking strength.

- Put forward a set of quantitative assessment index system for emergency capacity.

- Study the urban emergency capacity based on the index system and external DEA.

\section{THE URBAN ADAPTIVE STRENGTH OF DISAS- TER RELIEF}

\subsection{The Definition of Five-Dimensional Adaptive Strength}

The city's adaptive strength of disaster relief is a new research areas compare to traditional natural disaster evaluation, such as the assessment of disaster-making factors. The object of natural disaster risk assessment is every natural calamity, namely disaster-making factors, however the research object of city's adaptive strength is urban itself, namely all the constituent parts of a city. Through analyzing the mechanism of city relief, the paper proposed the concept of city mechanical performance, which is a research problem of mechanics of materials [14]. City mechanical performance is defined as innate mechanical performance when it is in the face of incident. So fivedimensional adaptive strength is proposed, which includes 
tensile strength, bending strength, abrasion resistance, fatigue strength, impact strength and breaking strength.

\subsubsection{Tensile Strength}

Tensile strength represents the maximum tensile forces per unit area that the material can offer during tensile process. However it is defined as the maximum tensile stress in urban emergency when it faces the physical destructions of an incident. For example, it can reflect the wind-resistance of public installation when a city is hit by a typhoon. The basic model of tensile strength is as follows:

$\sigma_{\mathrm{b}}=\beta^{*} \mathrm{P}_{\mathrm{b}} / \mathrm{A}_{0}$

$\sigma_{b}$ represents tensile strength of a city. $P_{b}$ is the grade of an urban disaster, such as force 12 winds. $A_{0}$ describes the disaster area of stricken city. $\beta$ is the transform coefficient.

\subsubsection{Bending Strength}

Compare to the ability of material bending resistance in mechanics of materials, it describe the capacity to keep the city function when it is destroyed by some disasters in urban emergency. For instance, the grade of urban bending strength can represent the city's ability to resist earthquake. The building in Japan can stand in the eight-magnitude earthquake; however a five-point-eight-magnitude earthquake can destroy most of the house in Yunnan province, China. Bending Strength cannot be measured directly, but we can use computer simulation technology to imitate this strength.

\subsubsection{Abrasion Resistance}

There is not the corresponding concept of abrasion resistance in mechanics of materials; it is the ability to resist the same degrades disaster after successfully responding an incident in urban disaster, especial in the old city. For example, the drainage system in Paris has existed for more than several hundred years, which can still face the Flooding Disaster. But the inadequate drains in Jinan Shandong Province, China did not stand in a couple of hours' Heavy rainfall in 2007; nearly 100 persons were drowned in the heavy rainstorm. So we can give a subjective judgment that the abrasion resistance in Paris is higher than it in Jinan. Besides, the expert in the field of city planning can choose the highdegrade abrasion resistance material to build the overdelicate facilities base on the experimental means and methods.

\subsubsection{Fatigue Strength}

Fatigue strength represents the maximum stress that engineering materials can still work after shocked by this force several times. So we can define the fatigue strength of urban emergency as the functional ability of a unban system when it suffer from a series of disaster. The basic model of tensile strength is as follows:

$$
\sigma_{\gamma}==\beta * P_{b} * \gamma / A_{0}
$$

$\sigma_{\gamma}$ is the tensile strength of a city. $P_{b}$ represents the grade of an urban disaster. $A_{0}$ describes the disaster area of the stricken city. $\beta$ is the transform coefficient.

\subsubsection{Breaking Strength}

Breaking strength of a unban system is the ability of city facility in the process of resisting unstable disaster crack extension, which is mainly used to measures the vulnerability in mechanics of materials. Although it describes the disaster impact in the same direction, its object in the process of evaluation mainly includes unstable urban system.

\subsection{The Comprehensive Urban Adaptive Strength}

All the 5 levels' urban adaptive strength can be analyzing from the physical properties of a city, but we cannot use this degrade to evaluate the urban emergency capacity and plan of the city directly. So the comprehensive strength of a city was proposed, which integrates the five-dimensional strength to the comprehensive urban adaptive strength in the uniform port criterion. This method has been used in emergency management for many years, for example we use fourdegrade to describe the likely destruction of nearly all disaster in China.

Generally speaking, we can define the comprehensive strength as the analytic function of the five-dimensional, namely the urban comprehensive strength $=F$ (tensile strength, bending strength, abrasion resistance, fatigue strength and breaking strength). Because of the difference of urban location and climate, the weight of the single strength is different. For instance, the common disaster in Taipei is typhoon and earthquake, so we should put on the weight of tensile strength and bending strength when build or improve the model; the dust storm and drought in Egypt require increasing the weight of abrasion resistance and fatigue strength. Therefore we choose the weight of the comprehensive model that is based on the expert or professional advice; and the common mathematical methods include AHP and Delphi.

The comprehensive strength defined by the above method has some limitations, which can only represent the static ability to deal with the disaster. However the weight of strength is dynamic in the process of urban emergency management. For example, when a category 5 storm occurs, the urban tensile strength will decrease. In order to reflect the dynamics and uncertainty of disaster, fuzzy mathematics theory should be added to the original model. The thought on fuzzy theory will be introduce in section 3.3.

\section{A SET OF WHOLE LIFE CYCLE ASSESSMENT INDEX SYSTEM FOR URBAN EMERGENCY CA- PACITY}

The assessment in emergency management is a very complex and important work. It is not only the base of prevention, rescue and management among the incident response, but also the basis in scientific decision-making. So the urban risk assessment index system should face to the whole life cycle of the emergency.

\subsection{The Thought of Whole Life Cycle Emergency As- sessment}

The whole life cycle of urban emergency has two meanings, and its narrower concept mainly includes information acquisition, effective response, major disposition and fast recovery. In a generalized process, there are the stages of forecasting, warming, risk identification and risk assessment before the narrower concept. As shown in Fig. (2). 


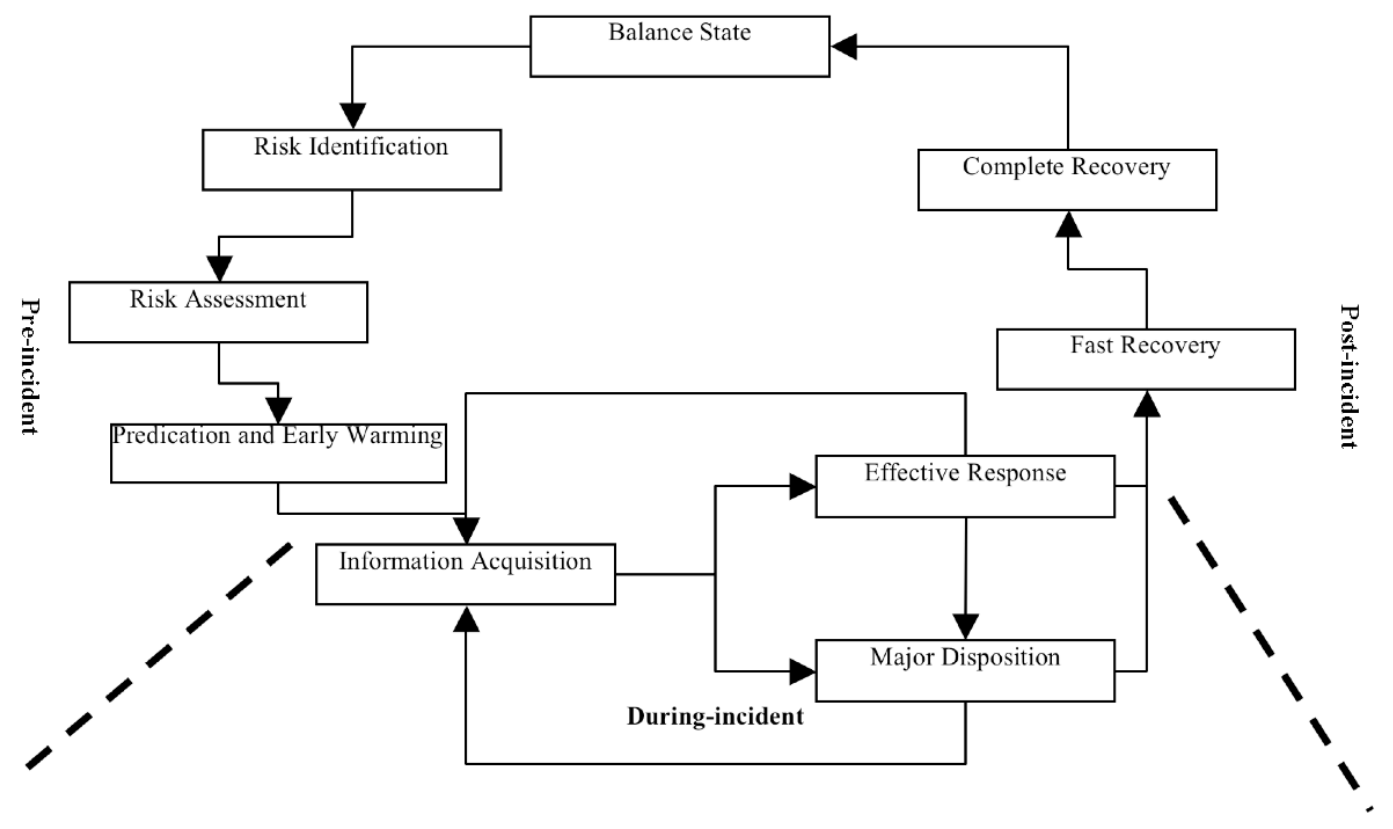

Fig. (2). Whole Life Cycle Emergency.

In time dimension, the assessment of urban emergency capacity can be divided into pre-incident assessment, duringincident assessment and post-incident assessment. Firstly, pre-incident assessment is the predictability evaluate of a incident's danger level, the analytic approaches mainly include the method of investigation, Delphi, Decision Trees, AHP, Fuzzy Comprehensive Assessment and Monte Carlo Simulation etc. Secondly, during-incident assessment the real-time evaluation to a happening incident, which can provide effective support for the decision, and which can be divided into Mitigability Measurement, Salvability Measurement, and Recoverability Measurement [15]. Finally, postincident assessment focuses on complete recovery and loss estimation.

The whole life cycle in this paper is the narrower explanation, namely during-incident assessment. There are not much research results in this field; the reason is that nearly every incident is dynamic and the absence of information. In order to have a comprehensive understanding of urban emergency, which can be described the thought of mitigability, salvability and recoverability. Mitigability is a measurement of incident defined as the degree or rank that an incident can be mitigated. Salvability is a priority assessment about the trapped objects in the process of rescuing the casualties and saving the property after the incident occurred. Recoverability is an infrastructureoriented assessment, measuring the difficulty level, time and resources in the process of turning back to the basic normal state after disaster.

\subsection{A Series Assessment Index of Urban Emergency Ca- pacity}

In order to deal with urban incident effectively, scholars usually study the mechanism of a special disaster, such as the principle of earthquake. But the most of disasters that we face to is uncertain; the paper attempts a general assessment method of urban emergency and relief. According to the construction of a city and the process of urban rescue, section 3.2 describe a series of index of urban emergency capacity, which includes urban vulnerability, institutional resilience, urban system rationality, urban system stability, urban plasticity.

\subsubsection{Urban Vulnerability}

The meaning of vulnerability differs from subject to subject, for example the vulnerability in the field of ecosystem is pressure and disturbance resulting from changes of environment. Urban vulnerability in this paper represents the capacity of keeping sustainable development after a series of natural disasters and social incidents.

As a complex evaluation index, it is difficult to measure community vulnerability. The paper divides it into several vulnerabilities from different dimensions, and then a comprehensive urban vulnerability can be described. Here is a rough evaluation of community vulnerability, region vulnerability index (RVI) is used to represent it. Although there are many factors which will affect the urban vulnerability, we just choose three main factors. $T$ hey include hazard vulnerability index (HazVI), behavior vulnerability index (BEVI) and social vulnerability index (SoVI). The urban vulnerability can be estimated with the weight sum of these main factors. The formulation is as follows:

$$
R V I=w_{1} H a z V I+w_{2} B E V I+w_{3} S o V I
$$

\subsubsection{Institutional Resilience}

Institutional resilience describes the institutional capacity of returning to the normal state after responding and dealing with incidents, which is a kind of representation for salvability. It contains resistive resilience and adaptive resilience in detail. Some scholars give a rough model to evaluate institutional resilience, which point out that institutional resilience index (RI) is the function of the rate 




Fig. (3). Assessment index system based on the certain idea.

of state guarantee $(\mathrm{S})$, recovery time $(\mathrm{T})$, the rate of resource supply (RS) and recovery level (RL). According to the Correlation analysis of these factors, the paper gives a simply model of institutional resilience. (Chen et al., 2009).

$$
R I=\frac{S \times R L}{T \times R S}=\frac{S}{R S} \times \frac{R L}{T}
$$

Ps. S/RS describes the contrast of resource between preincident and post-incident; $\mathrm{RL} / \mathrm{T}$ represents the recovery capacity of an institute.

\subsubsection{Urban System Rationality}

Urban System Rationality describe the effect of starting up emergency response, executing emergency preplan, building urban rescue centre, taking disaster scenario analysis, which focus on the analysis of salvability. According to the efficiency of emergency, we can divide rationality into 5 levels: perfect, reasonable, effective, confusion and inefficient. Five dummy variables are need to represent these level when the paper build index system in section 3.3.

\subsubsection{Urban System Stability}

Urban system stability stands for a kind of capacity to keep the original state after a series of disaster, which is the thought of recoverability. As a combine system of nature and society, its stability is determined by many factors. Urban natural system $\left(\mathrm{ST}_{\mathrm{n}}\right)$ is relative stability in a long time, but urban social system is full of variety. Its effective factors include the development level of urban facilities (d), urban area (s), urban population $(\mathrm{N})$, urban vehicle fleet $(\mathrm{T})$, politics (p), economics (e) and culture (c) ect. According to the relationship of these factors, we can simply describe the urban system stability (ST).

$$
\mathrm{ST}=\mathrm{F}(\mathrm{d}, \mathrm{N} / \mathrm{s}, \mathrm{T} / \mathrm{s}, \mathrm{p}, \mathrm{e}, \mathrm{c}, \beta, \cdots)+\mathrm{ST}_{\mathrm{n}}
$$

Ps. N/s is the intensive degree of urban population; $T / s$ is the relative quantity of urban motor vehicles; $\beta$ is disturbance.

\subsubsection{Urban System Plasticity}

Urban system plasticity is the capacity that city can stand a series of plastic deformation of urban facilities during the process of handling incidents, which represents the thought of recoverability in modern emergency management. Earthquake, debris flow and flood etc. natural disasters require a relative high urban plasticity, for example, nearly every building will plastic deformation after a large earthquake. Because earthquake is unpredictable, we can improve the ant seismic level of buildings, which is a kind of urban plasticity. Urban system plasticity is determined by the material of urban facilities, so we can use the plasticity of facilities' material to stand for it, namely material percentage reduction of area $(\Psi)$.

$$
\Psi=\left(\mathrm{A}_{0}-\mathrm{A}_{1}\right) / \mathrm{A}_{0} \times 100 \%
$$

Ps. $A_{0}$ is cross section area before plastic deformation; $A_{1}$ is cross section area after plastic deformation.

\subsection{Assessment Index System of Urban Emergency Ca- pacity Based on the Idea of Fuzzy Mathematics}

The five-dimension assessment index of urban emergency capacity is affected by both city itself and outer circumstance, but urban adaptive capacity is an endogenetic factor that can determined these index. Section 3.3 gives three kinds of method to build the index system.

(1). Assessment index system of urban emergency capacity based on the certain idea is a kind of index system that is adopted by many cities. For instance, expert group on emergency usually set the urban emergency grade after analyzing the comprehensive urban adaptive strength and the disaster itself. The process is as Fig. (3).

(2) Assessment index system of urban emergency capacity based on the weighted average idea is hot in the field of emergency evaluation. The city disaster takes on some new characteristics, such as diversity and unconventional etc., so a single assessment index cannot reflect urban emergency capacity completely. Many scholars put forward a series of index from different aspects, for example, Zhao Ling and Tang Minkang (2008) proposed an index system that included guarantee ability of resource, ability of disaster rescue, ability of emergency response etc. Although the $\mathrm{N}$-dimension assessment index is reasonable, we should integrate them into a comprehensive index system by the method of weighted average. The process is as Fig. (4).

(3) Assessment index system of urban emergency capacity based on Fuzzy Mathematics is proposed in the paper firstly. Urban is a complex system, and we cannot give a comprehensive measure to evaluate its emergency capacity completely. A so called comprehensive index system can mislead the urban emergency planning, for instance, because of the robust urban facilities of Guangzhou compare to a small county in Liaoning province, we will evaluate that the emergency capacity in Guangzhou is higher than the small 


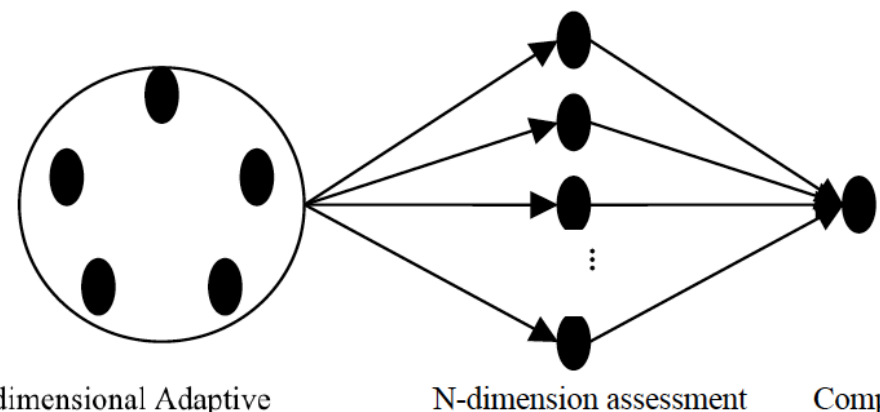

Five-dimensional Adaptive

$\mathrm{N}$-dimension assessment

Comprehensive index

Fig. (4). Assessment index system based on the weighted average idea.

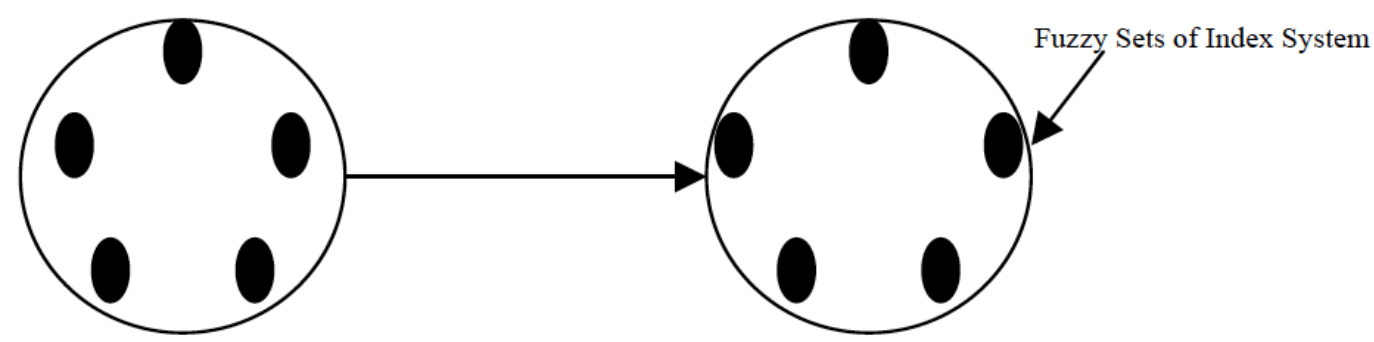

Five-dimensional Adaptive Strength

Fig. (5). Assessment index system based on fuzzy mathematics.

county base on the weighted average idea. But the 2008 snow hazard made the urban paralyzed, which is considered a medium snow in Liaoning, we should not determined the urban emergency capacity on the certain idea. The idea of Fuzzy Logic and Fuzzy Mathematics is a new way to solve the contradiction [16]. The mapping relation is as shown in Fig. (5).

\section{A THEORETICAL MAGIC CUBE MODEL OF UR- BAN EMERGENCY CAPACITY BASED ON EXTER- NAL DEA}

The urban emergency system is just like a production system, the increase of urban emergency input can affect the capacity to resist disasters. So this paper will conduct an exploratory model to evaluate emergency ability, which is based on external DEA and is represented by the form of magic cube model. After getting or analyzing quantitative value of the index system in section 3.2, urban planners can use the Win4DEAP software to gain the capacity on the pattern of CCR- I and BCC-I.

\subsection{The brief introduction of DEA}

We usually meet a series of assessment problem in social activities, which is to evaluate decision-making units on its input indexes and output indexes. Input dates (such as the investment) are factors that can transform into output dates (such as the economic returns and social benefits) through the DEA model.

Data Envelopment Analysis (DEA) is proposed by American mathematicians in 1978. It is an assessment technique effective method to carry out compare relative efficiency and benefit for decision unit which has much input and much output aspects. The typical DEA models include CCR model, BCC model, CCGSS model, CCW model and CCWH model [17]. Because of its characteristics of facing to multi-input and multi-output dates, DEA has many opportunities to deal with urban emergency assessment which concern different levels of indexes. The advantages of DEA in dealing with urban disaster relief capacity are as follows in detail: DEA has the property of dealing dates indirectly, so the dimension of indexes is unimportant; there is no hypothesis of weight in input index, which can subjective factors. In DEA method, input index need not be related to a special output index, which meets the requirements of fuzzy assessment index system of urban emergency capacity in section 3.3. A typical DEA model of urban emergency capacity can be described in Fig. (6).

Fig. (6) describes an urban emergency assessment model with n-input index and m-output index. Here is a hypothesis that there are $\mathrm{K}$ decision making units $(D M U), \mathrm{K}>2(\mathrm{~N}+\mathrm{M})$, so we can get emergency effective index of every units. The first one is as follows:

$$
\operatorname{Max} T E_{0}=\frac{\sum_{m=1}^{M} \alpha_{m 0} Y_{m 0}}{\sum_{n=1}^{N} \beta_{n 0} X_{n 0}}
$$

$$
\begin{aligned}
& \text { s.t. } \frac{\sum_{m=1}^{M} \alpha_{m 0} Y_{m k}}{\sum_{n=1}^{N} \beta_{n 0} X_{n k}} \leq 1, k=1, \ldots, K \\
& \quad \alpha_{m 0}, \beta_{n 0} \geq 0, m=1, \ldots, M ; n=1, \ldots, N
\end{aligned}
$$




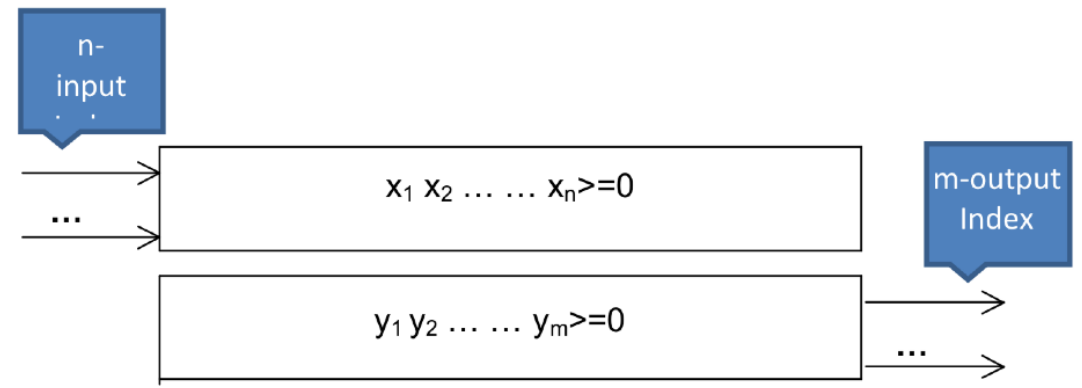

Fig. (6). A typical dea model of urban emergency capacity.

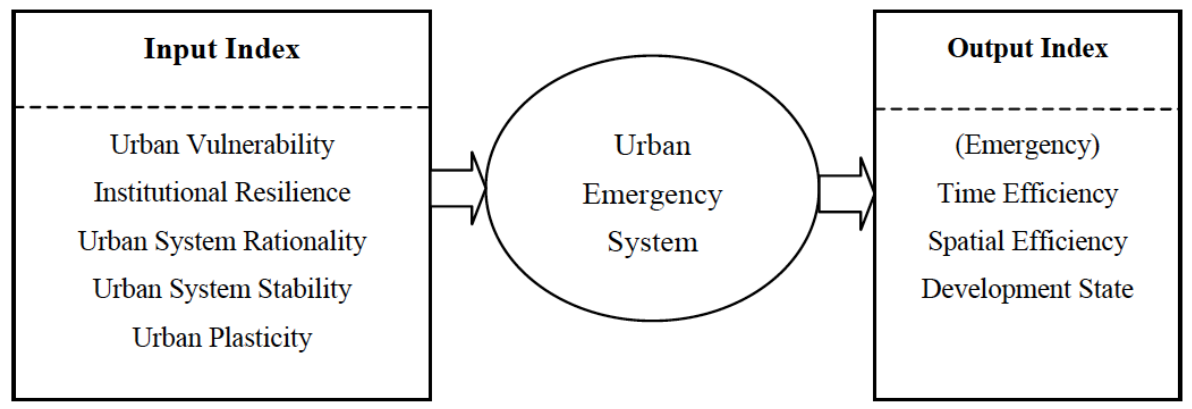

Fig. (7). Input and output indexes of urban emergency system.

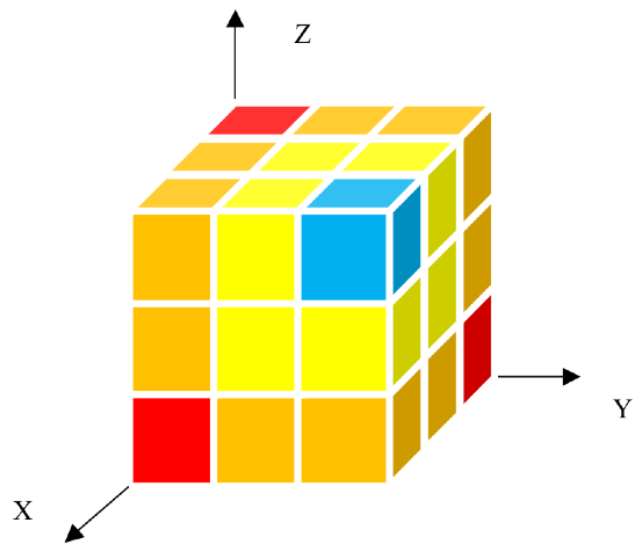

Fig. (8). The magic cube of urban emergency capacity.

Ps. the weight of input and output index is $\beta_{n 0}$ and $\alpha_{m 0}$. MaxTE0 represents the urban emergency effective index. $\mathrm{Xnk}$ and Ymk is the dates of $\beta_{n 0}$ and $\alpha_{m 0}$. There are $\mathrm{N}$ input indexes and $\mathrm{M}$ output indexes. The above formula is a monotonic function, which means the increase of input index cannot lead to the decrease of output index. It fays in with a rule of urban emergency.

\subsection{A Theoretical Model of Urban Emergency Capacity Based on External DEA}

In order to evaluate urban emergency capacity completely, input and output indexes should reflect urban disasters resistance ability from different aspects. The theoretical model in this paper introduces five input indexes and three output indexes. As is shown in Fig. (7).

In the above model, five-dimension input index is the mapping of urban adaptive strength in the field of urban emergency planning. Section 3.2 in this paper has given the connotation and measure method of these indexes. The output index in the model is based on emergency scenario analysis. Emergency time efficiency represents the speed of urban emergency in temporal dimension. Spatial efficiency describes the urban disaster resistance ability in spatial dimensions, which includes the allocation of urban fire station, the location of emergency resources of materials and the distribution of urban emergency shelter sites. Urban development state is a generalized concept, which expresses the social characteristics of urban system, such as politics, economics and culture etc. All the indexes above are the decisive factors of urban emergency capacity, but they are not isolated. There is a fuzzy and mutual interaction between them.

Through dividing the index into three parts, we can get 27 levels of urban emergency capacity. As shown in Fig. (8), the paper uses four colors to describe these levels, which is usually applied in crisis warming. Red represent the lowest urban emergency capacity, orange is higher than red. Yellow is the second grade and blue stand for the highest. The vivid expression form makes it convenient for scholars to evaluate urban emergency ability.

\section{CONCLUSION}

In previous sections, the urban adaptive strength and fuzzy assessment index system is defined and built. According to these concepts, the paper exploringly constructs a theoretical model of urban emergency capacity based on external DEA and the form of magic cube. There are some drawbacks in the model, for example the urban social factor is complex, which involves sociology and psychology. The next work will use incomplete information system to analyze the index. 


\section{CONFLICT OF INTEREST}

The authors confirm that this article content has no conflict of interest.

\section{ACKNOWLEDGEMENTS}

The authors thank the editor for helpful suggestions. This project is funded with support from National Basic Research Program of China (973 Program,2012CB955804), Foundation of the Education Department of Henan Province (14B170006), National Natural Science Foundation of China(41171438).

\section{REFERENCES}

[1] A. S. Markowski, "Fuzzy risk matrix", Journal of Hazardous Materials, vol. 159 pp. 152-157, 2008.

[2] Q. Zhou, W. L. Huang, and Y. Zhang, "Identifying critical success factors in emergency management using a fuzzy dematel method", Safety Science, vol. 49, pp. 243-252, 2011.

[3] H.H. Ni, A. Chen, and N. Chen, "Some extensions on risk matrix approach", Safety Science, vol. 48, pp. 1269-1278, 2010.

[4] Y. B. Tie, C. Tang, and C.H. Zhou, "The application of AHP to emergency response capability assessment in urban disaster", Journal of Geological Hazards and Environment Preservation, vol. 16, no. 4, pp. 433-437, 2005. (in Chinese).

[5] X. Wang, S. H. Wu, and M. M. Tawana, "An evaluation on urban emergency response capability against disaster based on ahpmethod", Shanxi Energy and Conservation, vol. 52, no. 1, pp. 4246, 2009. (in Chinese).

[6] G H. Chen, T. Liang, and H.W. Zhang, "Study on the methodology for evaluating urban and regional disaster carrying capacity and its application”, Safety Science, vol. 47, pp. 50-58, 2009.
[7] R. Francis, and B. Bekera, "A metric and frameworks for resilience analysis of engineered and infrastructure systems", Reliability Engineering \& System Safety, vol. 121, pp. 90-103, 2014.

[8] Y. L. Tian, and Q. Yang, "Urban disaster response capacity evaluation index system model design based on AHP-DELPHI method", Journal of Wuhan University of Technology (Transportation Science\& Engineering), vol. 32, no. 1, pp. 168171, 2008. (in Chinese).

[9] L. Zhao, and M. K. Tang, "Capability evaluation of emergency system against urban disasters", Occupational Health \& Emergency Rescue, vol. 26, no. 1, pp. 31-33, 2008. (in Chinese).

[10] M. Garcés, V. Villanueva, J. A. Mauri, A. Suller, C. García, and J. Lopez, "Factors influencing response to intravenous lacosamide in emergency situations: LACO-IV study", Epilepsy \& Behavior, vol. 36, pp. 144-152, 2014

[11] J Cheng, and J P Tao, "Fuzzy comprehensive evaluation of drought vulnerability based on the analytic hierarchy process -----an empirical study from Xiaogan city in Hubei province", Agriculture and Agricultural Science Procedia, vol. 1, pp. 126-135, 2010.

[12] K. Tanaka, "The impact of disaster education on public preparation and mitigation for earthquakes: a cross-country comparison between Fukui, Japan and the San Francisco Bay Area, California, USA, Applied Geography, vol. 25, pp. 201-225, 2005.

[13] X. Y. Wu, and J. H. Gu, "Advance in research on urban emergency management capability assessment at home and abroad", Journal of Natural Disasters, vol. 16, pp. 109-114, 2007.

[14] K. N. Sun, "Material for modern engineering and fundamentals of machine manufacturing technology", Academic Press, Beijing, 2005.

[15] A. Chen, N. Chen, and H. H. Ni, "Modern emergency management: theories and methods", Science Press Beijing, 2009.

[16] L. Běhounek, and P. Cintula, "From fuzzy logic to fuzzy mathematics: A methodological manifesto", Fuzzy Sets and Systems, vol. 157, no. 5, pp. 642-646, 2006.

[17] Q. L. Wei, "Data envelopment analysis", Science Press, 2004.

Received: September 16, 2014

Revised: December 23, 2014

Accepted: December 31, 2014

(C) Lu et al.; Licensee Bentham Open.

This is an open access article licensed under the terms of the (https://creativecommons.org/licenses/by/4.0/legalcode), which permits unrestricted, noncommercial use, distribution and reproduction in any medium, provided the work is properly cited. 